\title{
Colour and design: From natural patterns to monochrome compositions
}

\author{
Nilgün Olguntürk*, Halime Demirkan \\ Department of Interior Architecture and Environmental Design, Faculty of Art, Design and Architecture, Bilkent University, 06800 Bilkent, Ankara, Turkey
}

\section{A R T I C L E I N F O}

\section{Keywords:}

Colour

Nature

Design

\begin{abstract}
A B S T R A C T
There is no doubt that nature provides endless inspiration to the world of design. In order to explore the role of colour in design, forty-two people were asked to first choose a pattern from nature, then to abstract this pattern into geometric shapes and finally to colour this pattern. All work done by the participants were statistically analysed to find out the effect of colour on design. Findings of the study suggest that colour in a pattern is the first principal component of design as a unifier whereas this is replaced with the number of shapes in one group in black and white patterns.
\end{abstract}

○ 2009 Elsevier Ltd. All rights reserved.

\section{Introduction}

Pure geometrical shapes are often thought of as forms that can only be made by man. Such shapes, however, are found in nature, where there is an enormous variety of patterns derived mostly from the complex applications of simple laws [1]. Gombrich in The Sense of Order explores patterns in nature and describes how the contrast between order and disorder found there alerts our perception [2]. He also stresses that the distinctive designs exhibited by the flora and the fauna of the world were evolved with certain visible patterns that are somehow advantageous to specific organisms [2].

A pattern is created whenever lines, shapes, forms, colours and values are repeated [3]. Pattern is a regular, rhythmic, endless repetition. Rhythm refers to any movement characterised by a recurrence of elements at regular or irregular intervals. Rhythm incorporates the fundamental notion of repetition as a device to organise forms. Repetition means doing something again and again. The eye has a tendency to follow repetition, and this tendency is what gives the feeling of movement to visual rhythm [3]. Architects, for example, may use repetition of certain spaces to achieve a sense of order [3]. In ancient Greek temples, the columns repeat and set up a rhythm. Some commonly played games, such as chess, monopoly and tic-tac-toe, form another example. These are based on the repetition of circular, rectangular or square shapes.

Repetition usually has a unifying effect in a design [3]. In a pattern, repetition is the essence of order, being achieved in a pattern by using grouping. Grouping means uniting things that belong together in accordance with the designer's intentions. Grouping may emphasize form, colour, size, texture or shape. The

\footnotetext{
* Corresponding author. Tel.: +90312 2902429; fax: +90312 2664127.

E-mail address: onilgun@bilkent.edu.tr (N. Olguntürk).
}

Opera House in Sydney, Australia, by Jorn Utzon, has natural shell forms grouped into a rhythmic unit. The grouping involves proximity, similar form, colour and texture of material [3].

When diverse elements are employed, grouping can be achieved by emphasizing their similarity or proximity. Similarity is about sameness, that is, the visual characteristics that elements share in common. Proximity is about closeness in terms of distance, that is, the closeness or proximity of elements to one another. Grouped organisations can encompass forms and spaces that are dissimilar and related only by proximity [3].

Like all design problems, composing a satisfactory pattern is a challenge because designers may find that they lack the foresight required to solve the problem without creating new obstacles elsewhere. The grouping that needs to be repeated in a pattern may disintegrate in a sequence [2]. A pattern should also have distinctiveness with adaptability. A generic design, in textile patterns, is that of the palmette. This has manifold versions variously described as 'pineapple' or 'artichoke' designs. It is also assimilated in 'pomegranate' or 'carnation' designs, which have the advantage of distinctiveness with adaptability, thus permitting change [2]. While the basic arrangement stays the same, the designer can still adjust the relationship between figure and ground to change the relative weights of the design.

Colour in design is a flexible and powerful tool. Colours may be viewed as a kind of language as they serve as tools of communication between humans and their surrounding [4,5]. Colour can be defined as 'an inherent property of all materials and surfaces including everything from light and paint to art, from aesthetics to functionality and as an inseparable element of design' [6]. Colours can be used to please the eye with their infinite combinations so that human response is to prefer one combination over another [7]. Certain colour combinations also attract more attention than others [8]. In design, colour may be used as an enhancement, to show something more than would be hidden from the eye in black-and-white expressions. 
The aim of this study is to find out the extent to which colour contributes to design. An experiment was conducted where participants were firstly asked to design an achromatic pattern inspired from nature and then to colour it. Colour was added therefore, as an additional layer of design. Participants assessed their achromatic designs, using colour to stress the visual aspects of those designs without losing their basic characteristics. Both the achromatic and the chromatic patterns were statistically analysed using the following design concepts, in order to understand the distinctive contribution of colour.

\section{Design concepts}

\subsection{Design elements}

Design elements are aspects of design that are important in creating a pattern. These design elements are shape, size, number, proportion and colour (Table 1, Figs. 1 and 2). Some of the elements involve sub-categories that further define certain design characters. For example, size is a design element, while width, length and area form its sub-categories or design characters.

Shape is the outline or contour of an element. Nature utilises many shapes, some of which can be simplified into variations of the cone, cube, cylinder, sphere and pyramid. Natural shapes can involve an infinite number of variations, while keeping a certain likeness in many ways. For example, snowflakes, when looked at with a magnifying glass, seem at first sight to be very different, yet are alike in many ways. They all display symmetry (sameness around the centre) and are hexagonal in shape, while possessing visual differences individually. In a design consisting of many elements, the eye will search for a shape, or in this study a group of shapes, that seem to give an origin, or place to begin. In an effort to understand it, the eye will then follow the design to an end point, which in this study is given by the edges of the paper. For this work, the participants were restricted to using regular geometric shapes in their patterns. The concept of regular geometrical shape stems from the circle and the infinite series of regular polygons able to be inscribed within it. Primary shapes are the most significant of the regular shapes, and these are: the circle, the triangle and the square [9]. In addition to the three primary shapes, the participants were allowed to use all the polygons (e.g. the pentagon and the hexagon), the rectangle and the ellipse.

Size shows how small or large is an element or a group of elements. It is the physical dimension, the magnitude of an element. In a pattern, the width and the length of one group affect the design, together with the area this group occupies in the design field. In this study, the design characters related to size are: the width of one group, the length of one group and the area occupied by one group (Section 3.3, 1, 2 and 3 for both patterns).

Number is the quantity of elements. In a pattern, the number of shapes in one group affects the design. In this study 'black-andwhite' pattern shapes could only be in a combination of black and white, whereas for 'colour' patterns, the shapes would be monochrome, together with grey, black or white, depending on

Table 1

Design concepts.

\begin{tabular}{ll}
\hline Design elements & Assembly of design elements \\
\hline Shape & Orientation \\
Size & Geometric relations \\
Number & Figure-ground relationship \\
Proportion & Level of complexity \\
Colour & Design tools \\
\hline
\end{tabular}

the preference of the designer. The design characters related to number are: number of black shapes, number of white shapes, total number of black-and-white shapes, number of colour shapes, number of black, white and grey shapes and total number of colours, black, white and grey shapes in one group (Section 3.3, 4, 5 and 6 for both patterns).

Proportion is the relationship of one part to another, more specifically between parts of elements with respect to magnitude, quantity, or degree. It is usually expressed as a numerical fraction. In a pattern, the proportioning ratio of one group as width/length may affect the design. In this study, the design character related to proportion is the proportioning ratio of one group as width/length (Section 3.3, 7 for both patterns).

Colour is a sensation produced by visible electromagnetic radiation (light) that stimulates receptors in our eyes. In order to add dimension and depth to a design, the basic principles of colour and its value (brightness) need to be understood [10]. Systems of colour description have been developed and vary according to the different fields of study. They usually involve three characteristics. The Commission International de l'Eclairage (CIE) produced an equilateral triangular colour map to show the largest range of reproducible colours in the visual spectrum called the 1976 chromaticity diagram which provides a good representation of colours in terms of hue and saturation (Fig. 3). The HSI, hue-saturation-intensity, triangle model is also effective in showing the three dimensions of colour (Fig. 4) [11].

HSI is possibly the most basic of the descriptive systems and Stasiek et al., emphasize that HSI values might closely approximate to the way in which humans perceive and interpret colour [11].

Returning to the question of the different fields of study:

- For colour image processing, the HSI is used [11],

- for producers and users of colour monitors, video cameras and computer graphics, the combination of the primary colours (red, green and blue-RGB) is used. Again Stasiek et al., show how hue, saturation and intensity may be calculated from the coordinates of the RGB colour cube [11],

- for the publishing industry, where the mixing of inks is the dominant factor, the combination of the subtractive primaries (cyan, magenta, yellow and black-CMYK) is used,

- for this design-related study, hue, saturation and brightness are used, where the term brightness corresponds with intensity. These dimensions are now described in detail.

When we say, 'colour' we are often talking about hue. It is the name of a colour and indicates whether a colour looks red, green, blue, yellow, orange, etc. In this study, this design character is specified as hue (Section 3.3\14 in coloured patterns).

Saturation is about a colour being vivid vs. dull or weak vs. strong. It is the purity or strength of a colour determined by the amount of pure hue in a colour. In computer colour image processing, the different saturations can be produced with the white content [11]. In designing patterns, the participants produced different saturations by choosing the right amount of pigment for the hue they were using. For saturated colours they were using more pigment, while for less saturated ones they were using less. In this study, the design character related to saturation is the number of different saturations used in the pattern (Section $3.3 \backslash 15$ in coloured patterns).

Brightness is about lightness and darkness. It is the dimension of colour by which emitted light is ordered continuously from light to dark in correlation with its intensity. Many animals and insects utilise brightness as a defence mechanism: by assuming colours in shades that are similar to their environment, they 


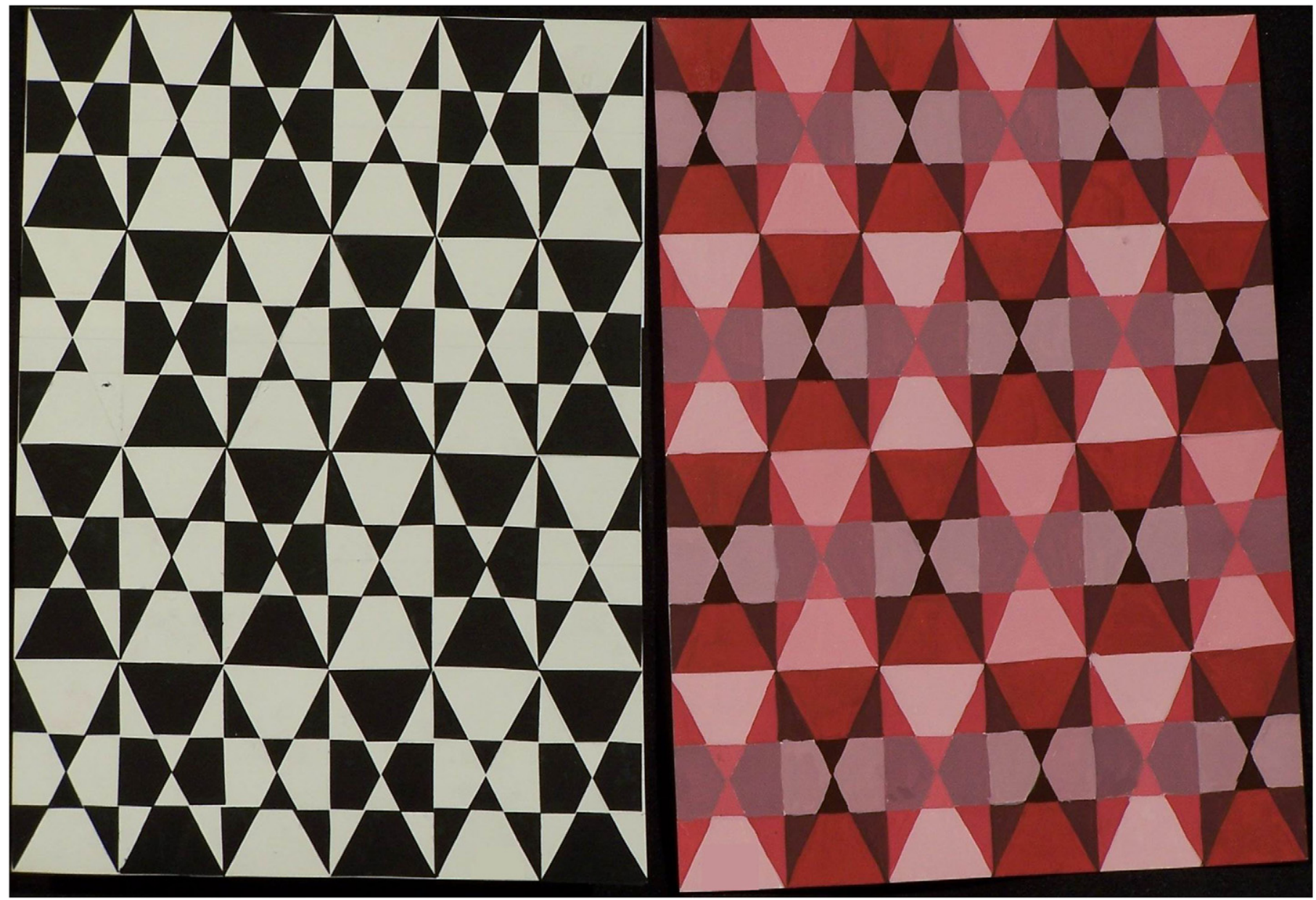

Fig. 1. Example of a pattern.

protect themselves against their natural predators. Most camouflage relies on hue, as well as brightness, but even the proper hue would not hide the animal unless the brightness is right, too [10]. Brightness, also commonly referred to as value, is inherent in any design. Even when there is no hue, there is brightness, which basically is the grey scale from black to white. Effective use of brightness in a design allows the viewer to see the design elements at their full potential [10]. Brightness can be used to establish contrast, in order to make shapes more obvious or to attract attention, or it can be used to blend the shapes into the background in a subtle way. If high contrast is used, there is a bolder statement. If subtle blending is used, there is softness either in light tones or dark tones [10]. In computer image processing, the different brightnesses can be produced with its grey-level (black-and-white) version of the image that is also known as intensity [11]. In designing patterns, the participants mixed a hue with black or white to produce different brightnesses on paper. The design character related to brightness is the number of different brightnesses used in the pattern (Section 3.3\16 in coloured patterns).

In this study, design characters related to colour are: the number of different colours, the number of black, white and greys, and the total number of colours, black, white and greys used in the pattern (Section 3.3 $\backslash 17,18$ and 19 in coloured patterns). As all coloured patterns were monochromatic, the number of different colours used in the pattern was all the different saturations and brightnesses of the chosen hue.

Colour harmony is the composition of colours that are aesthetically pleasing. To compose with colour means putting together two or more colours in such a way that they jointly produce an effect or an expression. Use of colour affects a design as it can influence the effect of any order by making the elements more or less conspicuous through contrast or brightness [2]. In this study, the black-and-white patterns were coloured with a monochromatic colour scheme (Figs. 1 and 2). A monochromatic colour scheme uses the harmony of similarities. It consists of using a single hue with many different brightnesses and saturations. A monochromatic colour scheme was used to limit the possibility of using an infinite number of hue combinations, so the hue variable was controlled and the data obtained could be grouped under broader titles.

\subsection{Assembly of design elements}

The way design elements come together, in other words, the assembly of design elements, is also important in creating patterns. Assembly of design elements can be achieved through orientation, geometric relations, figure-ground relationship, level of complexity and using design tools (Table 1).

Orientation is about direction. It is the direction of a group relative to the vertical viewing of the A4 paper in this study. The participants generally used triangles to indicate orientation in their designs. The orientations that participants used in this study are to the right, to the left (Fig. 2) and vertically down. Some participants also preferred to design patterns with balanced or central orientations (Fig. 1). In this study, the design character related to orientation is the orientation of one group with respect to the design field (Section 3.3 $\mid 8$ for both patterns). 


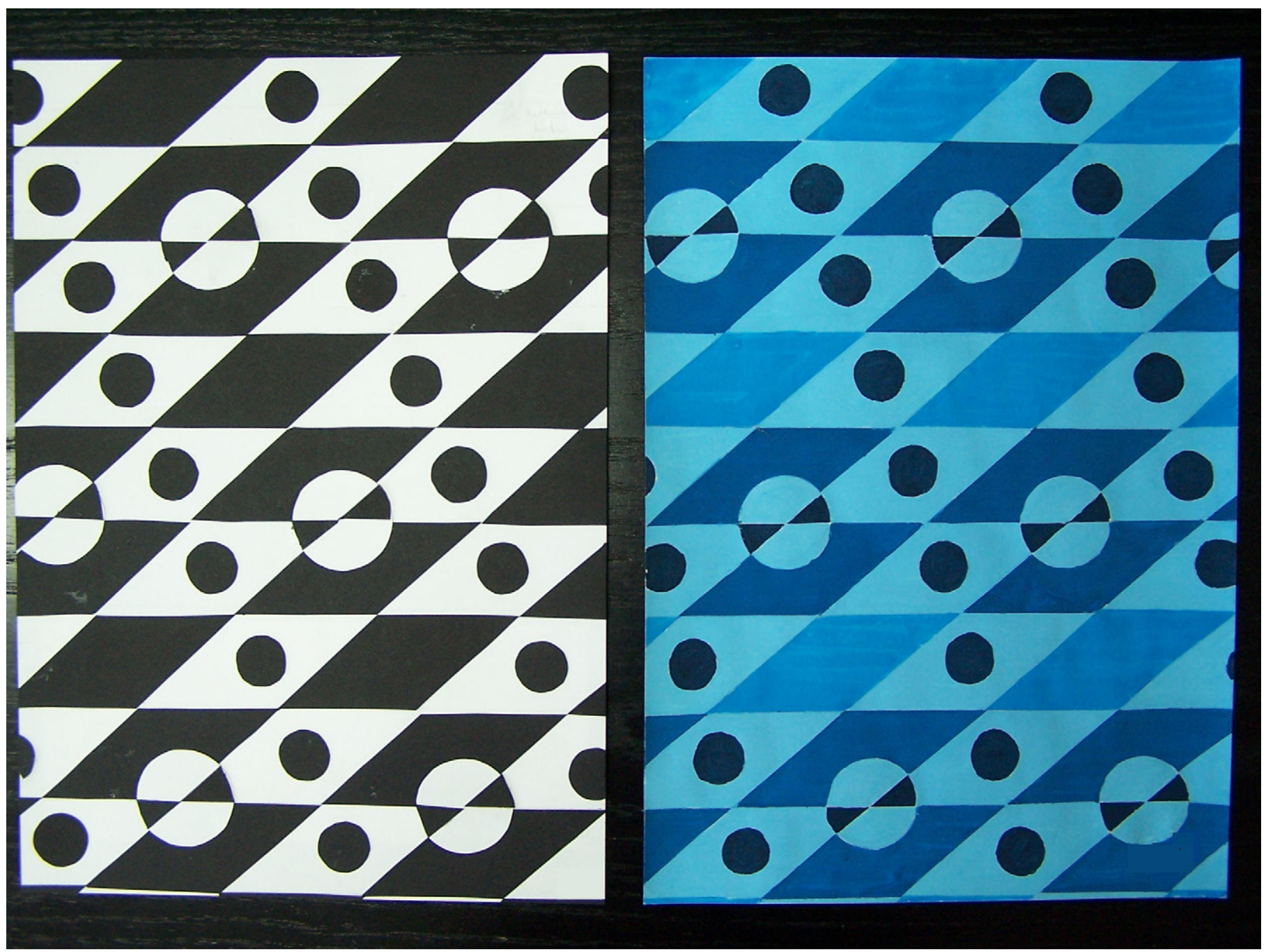

Fig. 2. Example of a pattern.

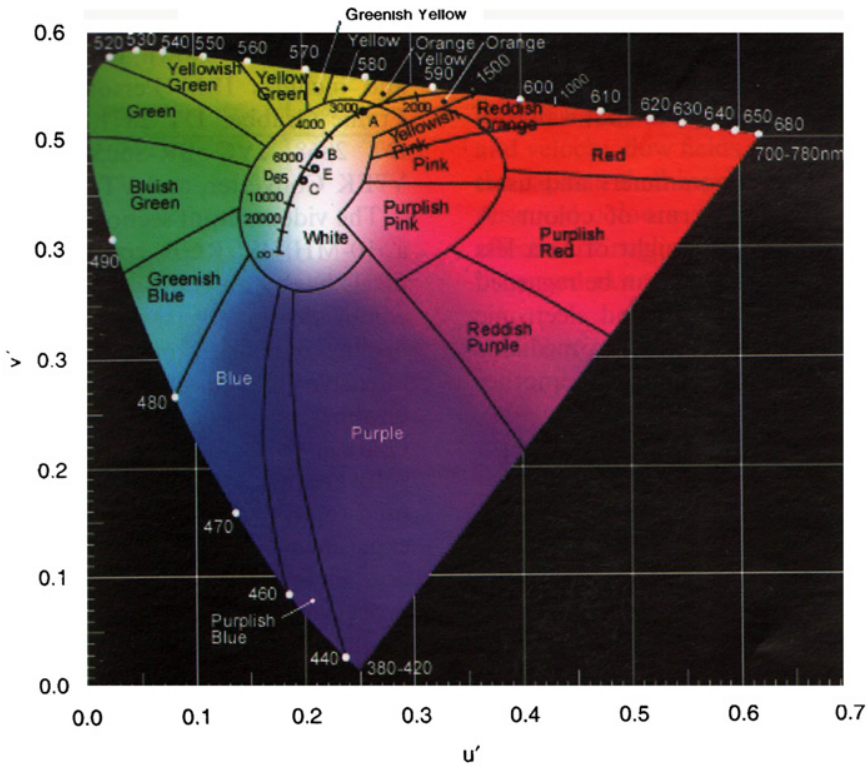

Fig. 3. The 1976 CIE chromaticity diagram.

Geometric relations are the way that geometries connect with each other. Different types of geometric relations may be used to establish better utilisation of the design field. Geometric relations

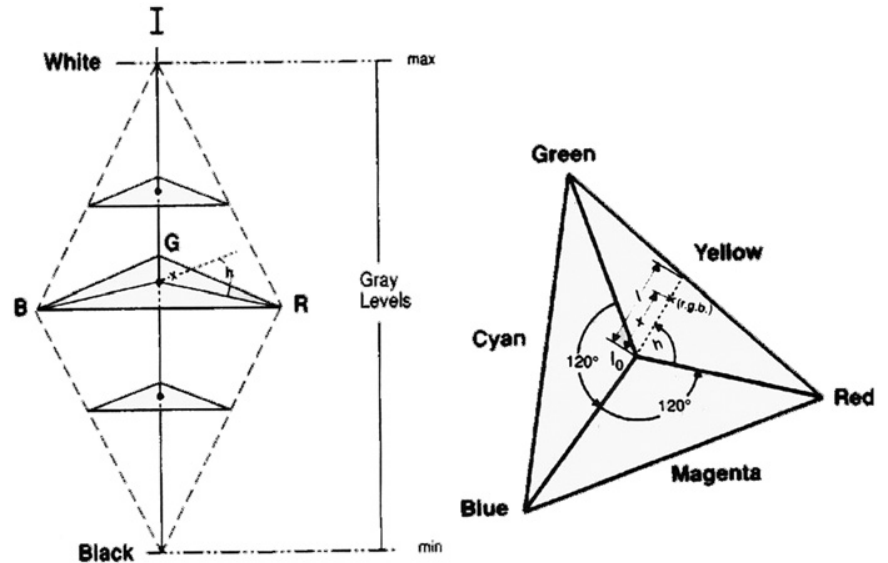

Fig. 4. The HSI triangle model.

that are used in the patterns are corner to corner (Figs. 1 and 2), corner to edge, edge to edge (Fig. 1), on top of (Figs. 1 and 2) and space between. In this study, the design character related to geometric relations is the type of geometric relations (Section 3.3 $\ 9$ for both patterns).

Figure-ground relationship is the inter-dependence between the elements of a design and the design field. In designing 
patterns, instead of a distinction between foreground and background, an unbroken sequence of shape and colour [12] is desired to be created. The correspondence between positive and negative shapes is given importance until figure and ground became integrated (Figs. 1 and 2). In this study, this design aspect is specified as figure-ground relationship (Section 3.3\10 in both patterns).

The level of complexity is also important in designing a pattern. In design, complexity is diversity or variety so that a visual interest can be created. Simple compositions can result in monotony or boredom, whereas complex compositions are prone to produce chaos if a strong order fails to be established. Thus, a successful amount of complexity with a readable order is necessary in designing a pattern. Complexity can be achieved in two ways: through the arrangement of shapes in one group and through the arrangement of groups within a design field (Figs. 1 and 2). The design characters related to complexity are simple vs. complex arrangement of shapes in one group and simple vs. complex arrangement of groups in the design field (Section 3.3 $\ 11$ and 12 in both patterns).

Design tools are the techniques for adding complexity to a pattern. By definition, a pattern requires the tools of repetition and rhythm to be used. In this study, the participants were free to use more design tools if they liked. The design tools used in the patterns created within this study are rotation, contrast, transparency, mirror image, translation, shape layering and shape generation. Rotation is a change in direction. Contrast is the opposition or dissimilarity of design elements. Contrasting elements are used to design with impact. Extremes give a design interest and keep it from being static or boring [10]. Contrast may be provided in size, colour, shape, number, texture and more. Contrast can be thought of as large or small, black or white, red or green, rounded or angular, one or many and smooth or rough. In the works of the participants, contrast in size and colour was most commonly used (Fig. 1). An illusion of transparency can be achieved with the geometric relation of collision (Fig. 2). Mirror image is using symmetrical groups. Translation is changing the orientation of one group with respect to another by moving one group in a direction other than the adjoining side. It is sliding one group of shapes with respect to another. Shape layering is repeating the shapes or a group of shapes with the geometric relation on top of/inside of. Shape generation is generating shapes by dividing/multiplying a shape that exists in the design or by adding a new shape to an already existing group. The design tools are used to alter an existing group and to repeat the existing group together with the altered group to create a pattern. This design character is specified as design tools (Section 3.3\13 in both patterns).

Figs. 1 and 2 show how all the previously described design elements come together to form a pattern. The pattern in Fig. 1 consists of the repetition of the group with two large triangles superimposed on three rectangles. The basic group has a central orientation with two triangles being directed towards the centre of the group. The arrangement of a single group is complex, because of the geometric relations selected to form the group. The arrangement of groups in the design field is simple as the one designed group is repeated over and over again on the whole design field, using the design tool of black and white or lightness and darkness contrast. The geometric relations used are on top of, when the two triangles come on top of the two side rectangles; edge to edge, with the rectangles coming together and corner to corner, with the two triangles coming together. The figure-ground relationship is integrated where the eye cannot differentiate between a separate figure and ground.

The pattern in Fig. 2 consists of the repetition of the group with three black and three white diamonds. Three small, black circles are on top of the white diamonds, and one white circle is made to collide with two of the black diamonds. The basic group has an orientation towards left, if the three black circles are accepted to be on the left of the black diamonds. The arrangement of a single group is complex, because of the geometric relations selected to form the group. The arrangement of groups in the design field is complex for the black-and-white pattern, as it is not easy to follow the beginning and the end of a single group. With the introduction of colour, a single group is made clearer to the eye, thus the arrangement of groups in the design field becomes simple. The design tool of transparency is used where the white circle collides with two of the black diamonds. The geometric relations used are on top of, where the small, black circles come on top of the white diamonds and corner to corner, with the way the diamonds come together. The figure-ground relationship is integrated where the eye cannot differentiate between a separate figure and ground.

\section{Method}

This study aims to find out how colour affects the design in comparison to other design concepts.

\subsection{Participants, the sample group}

There were 42 participants, all of whom were first year university students in an interior architecture and environmental design department.

\subsection{Phases of the experiment}

In the first phase, the participants were asked to study the natural environment to discover natural patterns. They were asked to bring an A4 photocopy of the natural pattern they would like to study further. The natural pattern needed to fill an entire A4 page with minimal white borders (top row, from left to right, all first images in Figs. 5-7).

In the second phase, the participants were asked to carefully study the structure, shapes and colour of their selected pattern. They were asked to find the structure and shapes in the natural patterns they chose (top row, from left to right, second images in Figs. 5 and 6). After this analysis, the participants were asked to design a pattern of regular geometries after the natural patterns they chose with cut and paste technique in black and white. They were expected to study the relationship of shapes in their natural pattern, regularise any inconsistencies and express these in a combination of geometric shapes while at the same time not losing the general characteristic of the natural pattern (top row, from left to right, third images in Figs. 5 and 6, second and third images in Fig. 7).

In the third phase, the participants were asked to use the geometric shapes that they had already designed in phase two to make a new pattern. They were free to change the sizes of geometric shapes, without changing their proportions. This exercise was also in black and white (bottom row, from left to right, all first images in Figs. 5-7).

In the fourth phase, the participants were asked to use a monochromatic colour scheme to further express the order and groupings in the new pattern that they had prepared in the third phase (bottom row, from left to right, all second images in Figs. 5-7). 


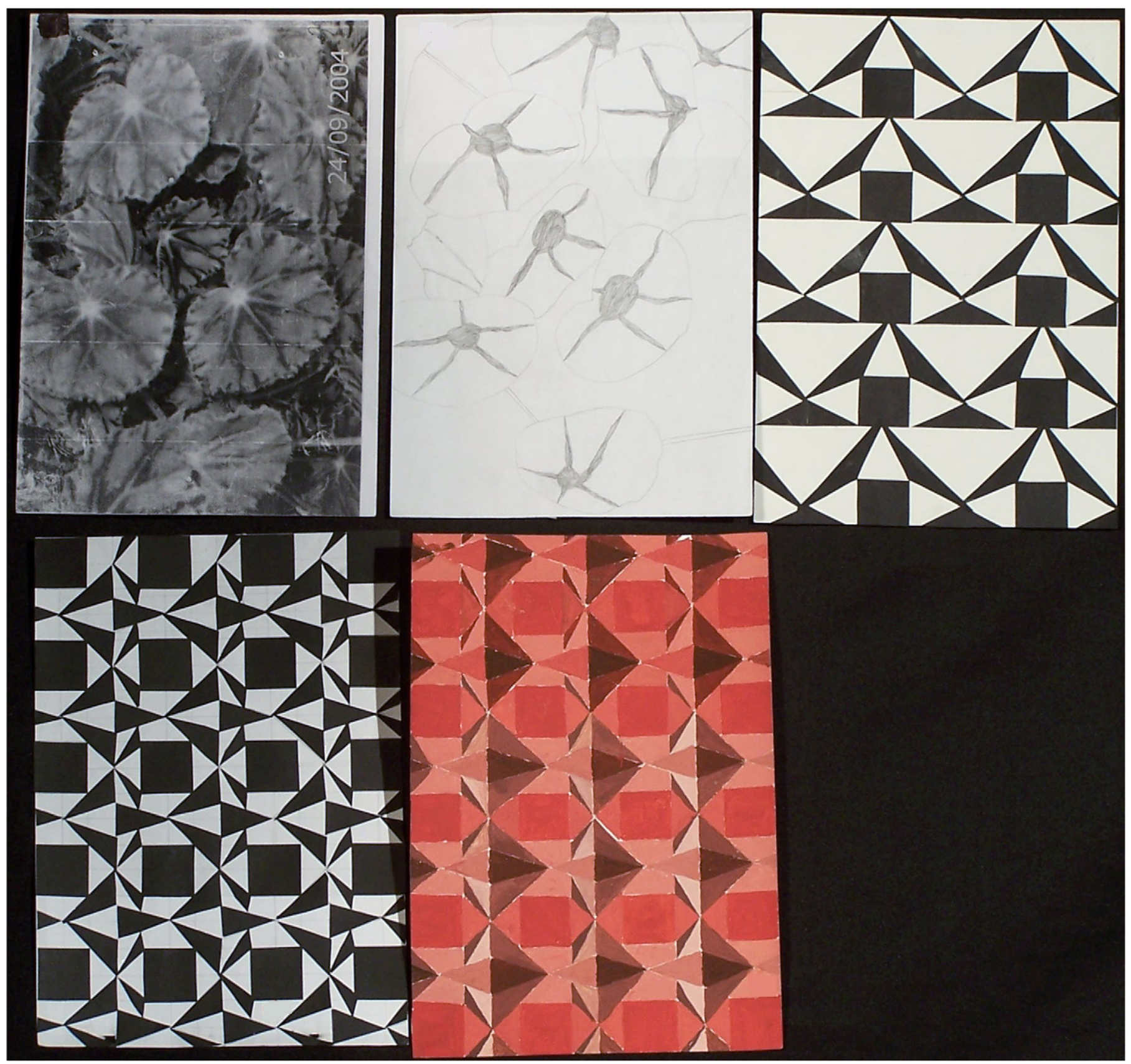

Fig. 5. A series of patterns derived from a group of leaves.

\subsection{Analysis}

Design concepts described in Section 2 are considered to be important for designing a pattern. The nature of design requires items that are countable, measured and have a distinct quality. Thus, design requires both quantitative and qualitative characters. Quantitative characters are measurable or countable and in this study they are either measured in centimetres or counted in numbers. Quantitative characters are all related to design elements. These are: width, length, area, number of shapes and proportion as described below: characters $1,2,3,4,5,6$ and 7 in both patterns and characters 15, 16, 17, 18 and 19 in coloured patterns.

Qualitative characters are not measurable, so they are brought together with a scoring system based on the assembly of design elements that are described in Section 2.2. These are: orientation, geometric relations, figure-ground relationship, complexity and design tools as below: characters $8,9,10,11,12$ and 13 in both patterns and character 14 in coloured patterns.

For black-and-white patterns the characters are specified as follows, with relative scores for each character indicated in parentheses:

1. Width of one group (size ranging from 2 to $20.7 \mathrm{~cm}$ );

2. Length of one group (size ranging from 1.6 to $11 \mathrm{~cm}$ );

3. Area occupied by one group (size ranging from 4 to $\left.196.7 \mathrm{~cm}^{2}\right)$;

4. Number of black shapes in one group (numbers: 1-13);

5. Number of white shapes in one group (numbers: 1-17);

6. Total number of black-and-white shapes in one group (numbers: 2-26); 


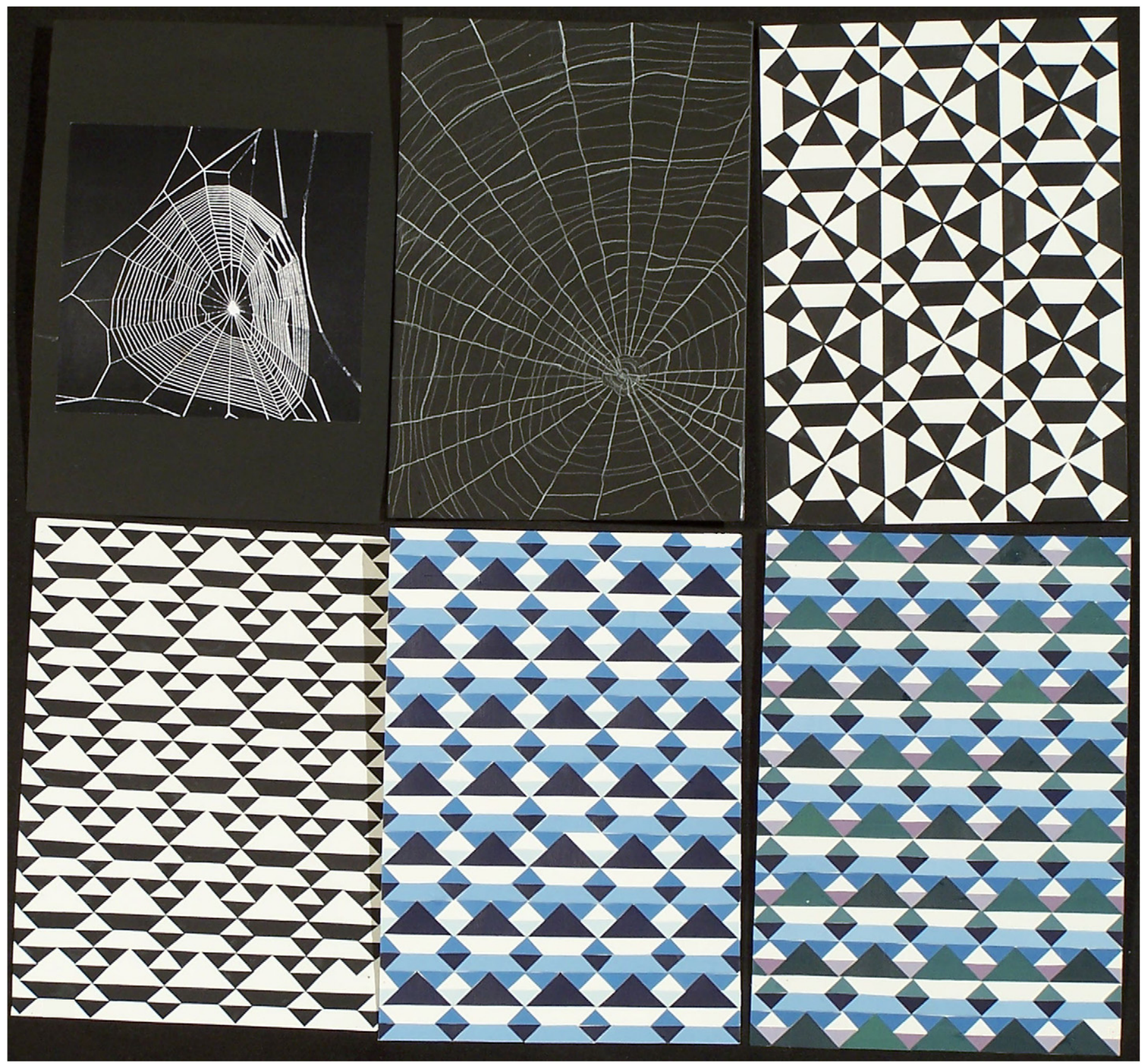

Fig. 6. A series of patterns derived from a spider web.

7. Proportioning ratio of one group as width/length (proportions: 0-6);

8. Orientation of one group with respect to the design field (1) central, (2) to the right, (3) vertically down, (4) to the left and (5) balanced;

9. Types of geometric relations (1) corner to corner, (2) corner to edge, (3) edge to edge, (4) on top of and (5) space between;

10. Figure-ground relationship (1) integrated and (2) not-integrated;

11. Simple vs. complex arrangement of shapes in one group (1) simple and (2) complex;

12. Simple vs. complex arrangement of groups in the design field (1) simple and (2) complex;

13. Design tools used to create the pattern (1) rotation, (2) contrast, (3) transparency, (4) mirror image, (5) translation, (6) shape layering, (7) shape generation and (8) none.
For colour patterns the same characters from the preceding black-and-white patterns are used, together with additional characters related to colour. Again, these are specified as follows, with relative scores for each character indicated in parentheses:

1. Width of one group (size ranging from 2 to $21 \mathrm{~cm}$ );

2. Length of one group (size ranging from 2 to $14.5 \mathrm{~cm}$ );

3. Area occupied by one group (size ranging from 4 to $210 \mathrm{~cm}^{2}$ );

4. Number of colour shapes in one group (numbers: 1-26);

5 . Number of black, white and grey shapes in one group (numbers: 0-17);

6. Total number of colours, black, white and grey shapes in one group (numbers: 2-26);

7. Proportioning ratio of one group as width/length (proportions: 1-3); 


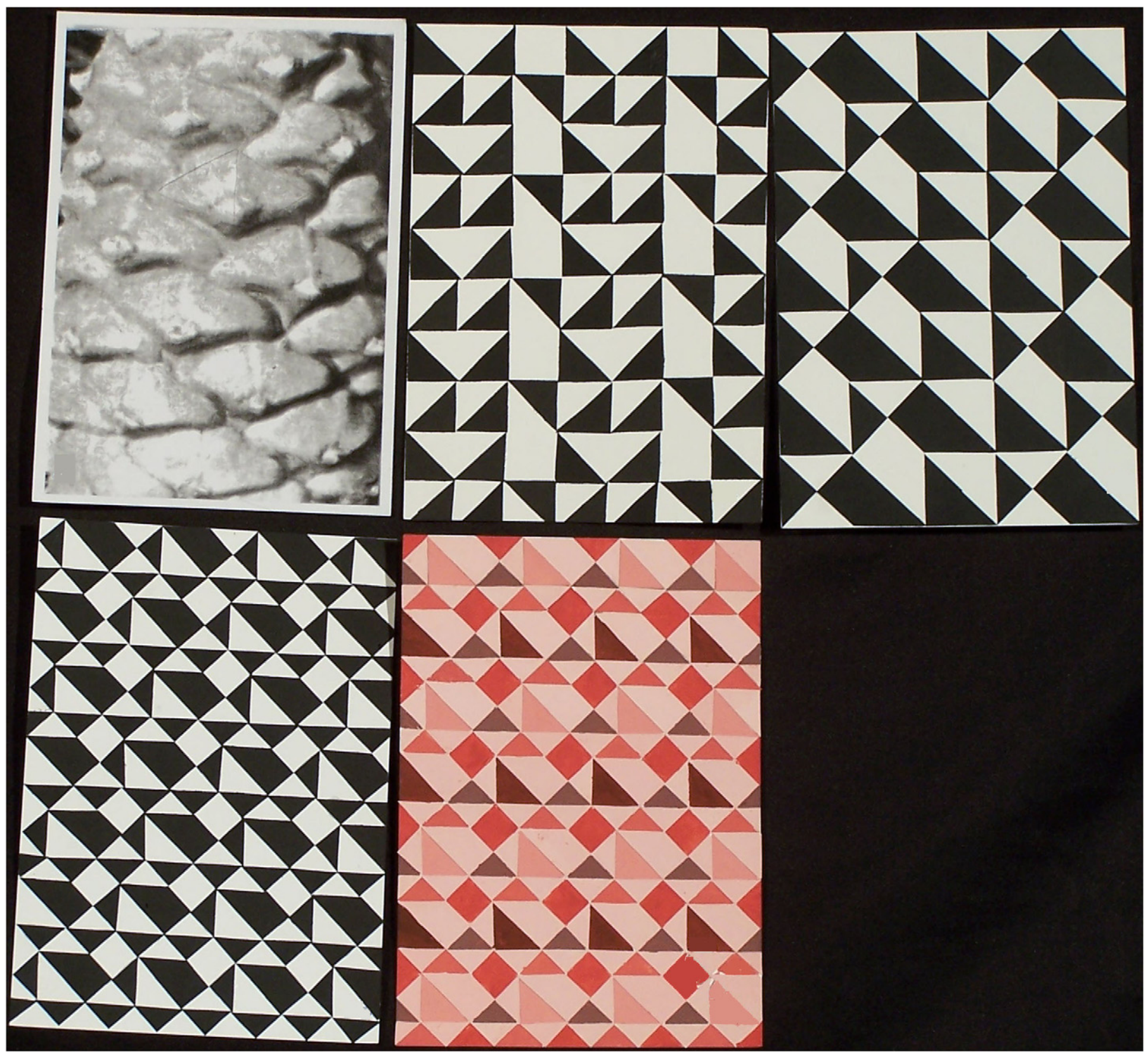

Fig. 7. A series of patterns derived from a pine cone.

8. Orientation of one group with respect to the design field (1) central, (2) to the right, (3) vertically down, (4) to the left and (5) balanced;

9. Types of geometric relations (1) corner to corner, (2) corner to edge, (3) edge to edge, (4) on top of and (5) space between;

10. Figure-ground relationship (1) integrated and (2) not-integrated;

11. Simple vs. complex arrangement of shapes in one group (1) simple and (2) complex;

12. Simple vs. complex arrangement of groups in the design field (1) simple and (2) complex;

13. Design tools used to create the pattern (1) rotation, (2) contrast, (3) transparency, (4) mirror image, (5) translation, (6) shape layering, (7) shape generation and (8) none;

14. Hue (1) blue, (2) red, (3) yellow, (4) green, (5) cyan, (6) magenta and (7) orange;
15. Number of different saturations used in the pattern (numbers: $1-8)$;

16. Number of different brightnesses used in the pattern (numbers: 1-6);

17. Number of different colours used in the pattern (numbers: 1-9);

18. Number of black, white and greys used in the pattern (numbers: 0-3);

19. Total number of colours, black, white and greys used in the pattern (numbers: 2-9).

Therefore, the Principal component analysis (PCA) was applied as a method for data reduction in order to classify the design concepts. Thus, it would be possible to determine how well the specified characters corresponded with the design concepts 
Table 2

Summary of total variance explained for black and white patterns.

\begin{tabular}{|c|c|c|c|c|c|}
\hline \multirow[t]{2}{*}{ Component } & \multirow[t]{2}{*}{ Eigenvalues } & \multicolumn{2}{|c|}{ Principal component analysis } & \multicolumn{2}{|c|}{ Varimax with Kaiser normalisation } \\
\hline & & Variance \% & Cumulative \% & Variance \% & Cumulative \% \\
\hline 1 & 4.29 & 33.02 & 33.02 & 23.84 & 23.84 \\
\hline 2 & 2.21 & 16.97 & 49.99 & 18.71 & 42.55 \\
\hline 3 & 1.90 & 14.58 & 64.57 & 14.99 & 57.54 \\
\hline 4 & 1.33 & 10.20 & 74.77 & 14.08 & 71.62 \\
\hline 5 & 1.20 & 9.24 & 84.01 & 12.39 & 84.01 \\
\hline
\end{tabular}

Table 3

Summary of total variance explained for coloured patterns.

\begin{tabular}{|c|c|c|c|c|c|}
\hline \multirow[t]{2}{*}{ Component } & \multirow[t]{2}{*}{ Eigenvalues } & \multicolumn{2}{|c|}{ Principal component analysis } & \multicolumn{2}{|c|}{ Varimax with Kaiser normalisation } \\
\hline & & Variance \% & Cumulative \% & Variance \% & Cumulative \% \\
\hline 1 & 5.13 & 26.98 & 26.98 & 19.85 & 19.85 \\
\hline 2 & 3.11 & 16.36 & 43.34 & 14.08 & 33.93 \\
\hline 3 & 1.99 & 10.49 & 53.83 & 13.80 & 47.73 \\
\hline 4 & 1.55 & 8.15 & 61.98 & 9.25 & 56.98 \\
\hline 5 & 1.33 & 6.98 & 68.96 & 9.00 & 65.98 \\
\hline 6 & 1.25 & 6.59 & 75.55 & 7.73 & 73.71 \\
\hline 7 & 1.07 & 5.62 & 81.17 & 7.46 & 81.17 \\
\hline
\end{tabular}
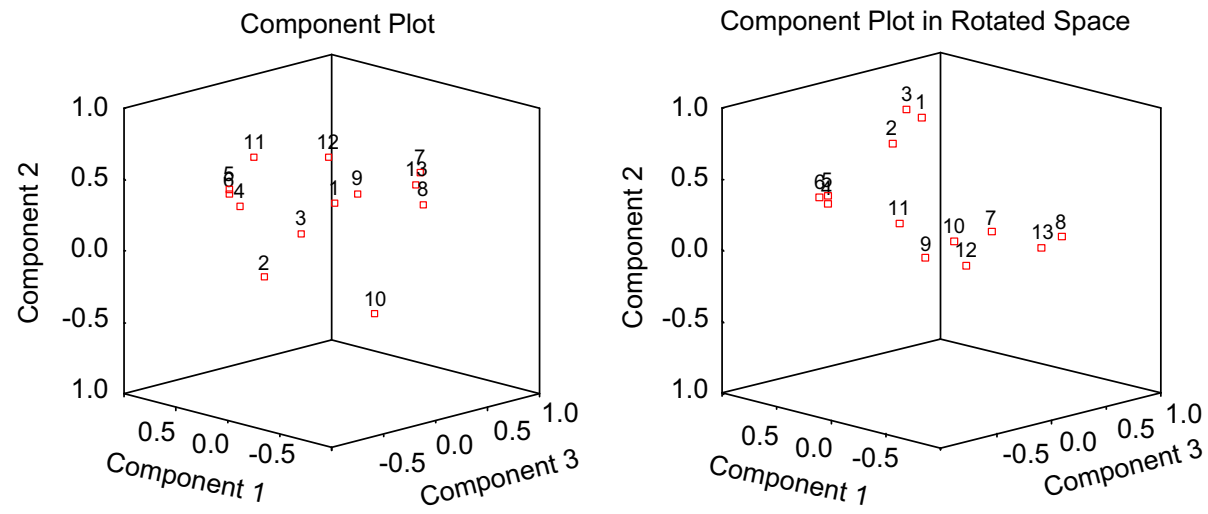

Fig. 8. Component plot of black-and-white patterns before and after rotation.

expected in the patterns designed. The PCA was applied to black and white, and colour designs separately in order to understand the contribution of colour to design.

The PCA method was used to determine the number of components with eigenvalues greater than 1.00 in all of the analyses with Statistical Package for the Social Sciences (SPSS). Table 2 shows that five components were initially extracted for black-and-white patterns. An eigenvalue of less than 1.00 was ignored since such components consist of uninterpretable error variation.

Table 3 shows that seven components were initially extracted for coloured patterns. Since there are more than two characters an orthogonal factor rotation was performed using the Varimax with Kaiser Normalisation in order to simplify components by maximising the variance of the loadings within components, across characters [13]. The varimax criterion maximizes the sum of the variances of the squared coefficients within each eigenvector and the rotated axes remain orthogonal.

\section{Results}

\subsection{Black-and-white patterns}

Using the PCA, five components were extracted. These five components were then orthogonally rotated using the Varimax with Kaiser Normalisation (Fig. 8) and the loadings of the 13 characters on the five components are shown in Table 2. In Table 4, the components with three or more characters that have loadings greater than 0.50 were chosen.

'Number in one group' was found to be the first principal component for designing these black and white patterns. The total number of shapes in one group, which is the sum of both black and white shapes, was found to be the most important (0.95), followed by the number of black shapes (0.91) and the number of white shapes (0.87).

The second principal component in designing the black and white patterns was found to be the 'size of one group'. Characters 
Table 4

Results related to the black and white patterns.

\begin{tabular}{|c|c|c|c|}
\hline Principal components & Characters & & Loadings \\
\hline \multirow[t]{3}{*}{ Number in one group } & 6 & Total number of black and white shapes in one group & 0.95 \\
\hline & 4 & Number of black shapes in one group & 0.91 \\
\hline & 5 & Number of white shapes in one group & 0.87 \\
\hline \multirow[t]{3}{*}{ Size of one group } & 3 & Area occupied by one group & 0.95 \\
\hline & 1 & Width of one group & 0.86 \\
\hline & 2 & Length of one group & 0.77 \\
\hline \multirow[t]{3}{*}{ Assembly of design elements } & 10 & Figure-ground relationship & -0.84 \\
\hline & 12 & Simple vs. complex arrangement of groups in the design field & 0.80 \\
\hline & 11 & Simple vs. complex arrangement of shapes in one group & 0.60 \\
\hline
\end{tabular}
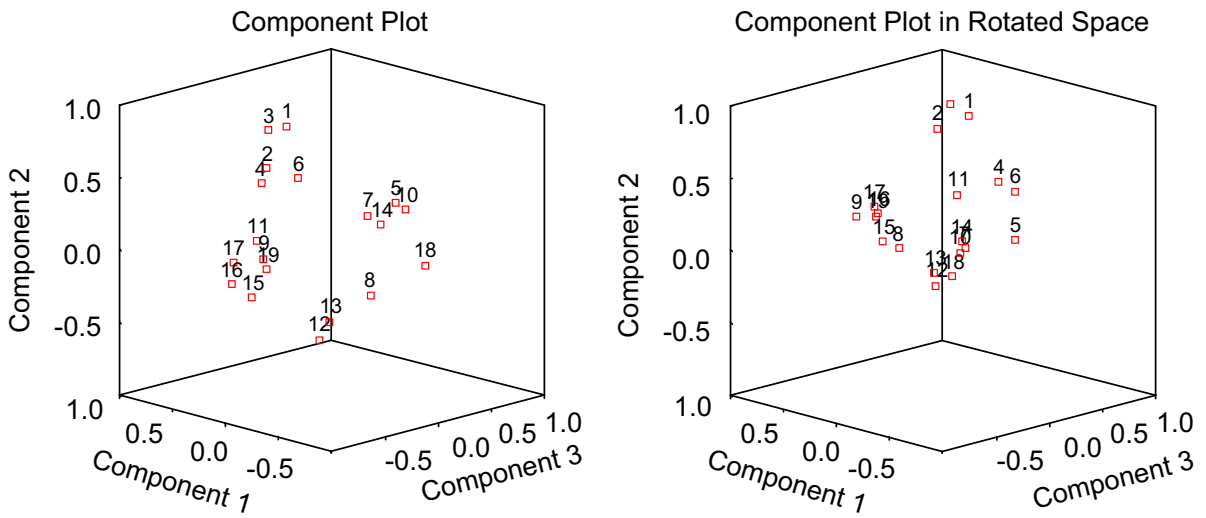

Fig. 9. Component plot of coloured patterns before and after rotation.

Table 5

Results related to the coloured patterns.

\begin{tabular}{|c|c|c|c|}
\hline Principal components & Characters & & Loadings \\
\hline \multirow[t]{5}{*}{ Colour in the pattern } & 17 & Number of different colours used in the pattern & 0.90 \\
\hline & 19 & Total number of colours, black, white and greys used in the pattern & 0.89 \\
\hline & 16 & Number of different brightnesses used in the pattern & 0.76 \\
\hline & 15 & Number of different saturations used in the pattern & 0.74 \\
\hline & 9 & Types of geometric relations & 0.73 \\
\hline \multirow[t]{3}{*}{ Size of one group } & 3 & Area occupied by one group & 0.97 \\
\hline & 1 & Width of one group & 0.88 \\
\hline & 2 & Length of one group & 0.81 \\
\hline \multirow[t]{4}{*}{ Number in one group } & 6 & Total number of colours, black, white and grey shapes in one group & 0.93 \\
\hline & 4 & Number of colour shapes in one group & 0.83 \\
\hline & 5 & Number of black, white and grey shapes in one group & 0.69 \\
\hline & 11 & Simple vs. complex arrangement of shapes in one group & 0.54 \\
\hline
\end{tabular}

under this principal component were the area occupied by one group (0.95), width of one group (0.86) and length of one group (0.77).

The third principal component that affects the design of blackand-white patterns was found to be the 'assembly of design elements'. Under this principal component, figure-ground relationship ( -0.84$)$, simple vs. complex arrangement of groups in the design field (0.80) and simple vs. complex arrangement of shapes in one group (0.60) were the characters that affected the design.

\subsection{Colour patterns}

Using the PCA, seven components were extracted. These seven components were then orthogonally rotated using the Varimax with Kaiser Normalisation (Fig. 9) and the loadings of 19 characters on the seven components are shown in Table 3. In Table 5, the components with three or more characters that have loadings greater than 0.50 were chosen.

The first principal component that affected the design of these colour patterns was found to be 'colour in the pattern'. Characters of colour were the number of different colours used in the pattern (0.90), the total number of colours, black, white and greys used in the pattern (0.89), number of different brightnesses used in the pattern (0.76), the number of different saturations used in the pattern (0.74) and types of geometric relations (0.73).

The second principal component was 'size of one group' with characters of area occupied by one group (0.97), width of one group (0.88) and length of one group (0.81).

The third principal component was 'number in one group'. Characters found were total number of colours, black, white, grey 
shapes in one group (0.93), number of colour shapes in one group (0.83), number of black, white and grey shapes in one group (0.69) and simple vs. complex arrangement of shapes in one group (0.54).

\section{Discussion}

The principal components affecting design and the importance of these components changed when colour was used. 'Colour in the pattern' became the first principal component when it was integrated into design (Table 5). Number of different colours used in the pattern was important to the success of the pattern (loading 0.90 , Table 5). The participants were asked to use monochromatic colour schemes, and the number of different colours ranged from one to nine different brightnesses and saturations of a single hue. Numbers of colours in the pattern together with achromatics were also important; these ranged from two to nine (loading 0.89 , Table 5). This suggests the importance of using achromatics together with colour in monochromatic colour schemes. The number of different brightnesses used in the pattern was also important; these ranged from one to six different brightnesses (loading 0.76 , Table 5). The number of different saturations used in the pattern was important as well; these ranged from one to eight different saturations (loading 0.74 , Table 5). These results indicate that the variety of colours used in the design is important in patterns. Hue, as a character on its own, was not loaded in the results. This does not show the unimportance of hue. This was most probably due to the monochromatic colour scheme used in the patterns, which did not offer a variety of hues to be used together. Another reason for this could be that our perception seems to be affected primarily by the brightness, followed by the saturation and lastly by the hue dimension of colour. This assessment was also suggested by the previous studies $[7,8]$. The hues that were preferred for designing patterns in this study were blue, red, yellow, green, cyan, magenta and orange. The PCA also grouped types of geometric relations together with colour, indicating a relationship between geometry and colour (loading 0.73, Table 5). Participants seemed to use colour in accordance with geometric relations.

In black-and-white patterns, 'number in one group' was the first principal component, which dropped to be the third principal component when colour was introduced (Tables 4 and 5). The total number of black-and-white shapes (loading 0.95, Table 4), the number of black shapes (loading 0.91, Table 4) and the number of white shapes (loading 0.87, Table 4 ) in one group all affected the design of black-and-white patterns. The total number of blackand-white shapes used in one group ranged between two and twenty-six, while the number of black shapes ranged between one and thirteen, and the number of white shapes ranged between one and seventeen (Section 3.3).

When colour was used, the total number of colour, black, white and grey shapes (loading 0.93, Table 5) and the number of colour shapes (loading 0.83 , Table 5 ) also became important, together with the number of black, white and grey shapes (loading 0.69 , Table 5) in one group. These results also emphasize the importance of using hues together with achromatics in these patterns. The number of shapes, regardless of their being monochrome or achromatic ranged from two to twenty-six in coloured patterns (Section 3.3). The PCA also grouped simple vs. complex arrangement of shapes in one group together with number of shapes indicating a relationship between complexity and number of shapes (loading 0.54, Table 5). Participants seemed to use the number of shapes to add complexity while forming their groups.
In this study, characters of width, length and area were scored independently as each would affect different aspects of design. For example, width and length affect rhythm, while area affects balance. These characters are by definition correlated and the PCA supported this as all these characters were grouped together under the principal component, 'size of one group'. The rank of 'size of one group' stayed the same for both black and white, and colour patterns, as being the second principal component (Tables 4 and 5). The loadings within this principal component also showed similarities. The area occupied by one group had the highest loading (loading 0.95, Table 4; 0.97, Table 5). This was followed by the width of one group (loading 0.86 , Table $4 ; 0.88$, Table 5) and the length of one group (loading 0.77 , Table $4 ; 0.81$, Table 5).

'Assembly of design elements' (the third principal component) in black-and-white patterns lost its effect completely in colour patterns (Table 4). For black-and-white patterns, figure-ground relationship (loading -0.84 , Table 4 ) and simple vs. complex arrangement of groups in the design field (loading 0.80, Table 4) were important. Simple vs. complex arrangement of shapes in one group was important for both black and white, and coloured patterns, but its alliance changed. In black-and-white patterns this was grouped under the 'assembly of design elements' (loading 0.60 , Table 4 ), while in coloured patterns it was grouped under the 'number in one group' (loading 0.54, Table 5).

Design, by its nature, brings together both quantitative and qualitative design concepts and their related design characters. In designing patterns, the quantitative characters seem to affect the design more than the qualitative characters. In black-and-white patterns, out of the nine design characters grouped under three principal components, six characters were quantitative and three characters were qualitative (Table 4). Similarly, for coloured patterns, out of 12 characters grouped under three principal components; ten characters were quantitative, while only two characters were qualitative (Table 5 ).

\section{Conclusion}

The study has shown that colour affects the design more than other design concepts. Findings of the study suggest that colour affects the design field as a whole, as opposed to black-and-white patterns where the concentration is more on the individual groupings. The study has also shown that quantitative characters of design, the design elements, affect the design of patterns more than qualitative characters, the assembly of design elements.

\section{Acknowledgements}

The authors wish to thank Dr. Kağan Olguntürk, Department of Communication and Design, Bilkent University, Ankara for photographing the patterns for this publication. Basic Design instructors of the Department of Interior Architecture and Environmental Design between the years 2004 and 2008 at Bilkent University, Ankara are acknowledged for the valuable discussions on design concepts.

\section{References}

[1] Arnheim R. The dynamics of architectural form. Berkeley and Los Angeles: University of California Press; 1977 p. 215

[2] Gombrich EH. The sense of order: a study in the psychology of decorative art 2nd ed.. Ithaca, New York: Cornell University Press; 1984 p. 87, 117, 191.

[3] Winters NB. Architecture is elementary: visual thinking through architectural concepts. Salt Lake City: Peregrine Smith Books; 1986 p. 23-4, 69-70, 187-90, 205-6. 
[4] Hard A, Sivik L. A theory of colors in combination-a descriptive model related to the NCS color-order system. Color Research and Application 2001;26.1:4-28.

[5] Şahin Ekici E, Yener C, Camgöz N. Colour naming. Optics and Laser Technology 2006;38(4-6):466-85.

[6] Dalke H, Little J, Niemann E, Camgoz N, Steadman G, Hill S, et al. Colour and lighting in hospital design. Optics and Laser Technology 2006;38(4-6): 343-65 p. 343.

[7] Camgöz N, Yener C, Güvenç D. Effects of hue, saturation and brightness on preference. Color Research and Application 2002;27(3):199-207.

[8] Camgöz N, Yener C, Güvenç D. Effects of hue, saturation and brightness: part 2: attention. Color Research and Application 2004;29(1):20-8.
[9] Ching FDK. Architecture: form, space, and order, 2nd ed.. New York: Van Nostrand Reinhold; 1996 p. 38, 356.

[10] Peterson BL. Using design basics: to get creative results. Cincinnati: North Light Books; 1996 p. 38-9, 69.

[11] Stasiek J, Stasiek A, Jewartowski M, Collins MW. Liquid crystal thermography and true-colour digital image processing. Optics and Laser Technology 2006;38(4-6):243-56 p. 244-5.

[12] Arnheim R. Visual thinking. London: Faber and Faber Limited; 1969 p. 286.

[13] Tabachnick BG, Fidell LS. Using multivariate statistics, 3rd ed.. New York: Harper Collins; 1996. 